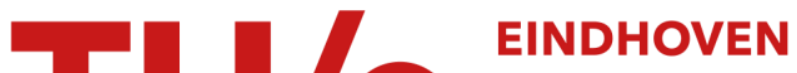 \\ UNIVERSITY OF \\ TECHNOLOGY
}

\section{The spectrum of an inhomogeneous membrane}

Citation for published version (APA):

Molenaar, J. (1990). The spectrum of an inhomogeneous membrane. (IWDE report; Vol. 9006). Technische Universiteit Eindhoven.

Document status and date:

Published: 01/01/1990

\section{Document Version:}

Publisher's PDF, also known as Version of Record (includes final page, issue and volume numbers)

\section{Please check the document version of this publication:}

- A submitted manuscript is the version of the article upon submission and before peer-review. There can be important differences between the submitted version and the official published version of record. People interested in the research are advised to contact the author for the final version of the publication, or visit the $\mathrm{DOI}$ to the publisher's website.

- The final author version and the galley proof are versions of the publication after peer review.

- The final published version features the final layout of the paper including the volume, issue and page numbers.

Link to publication

\section{General rights}

Copyright and moral rights for the publications made accessible in the public portal are retained by the authors and/or other copyright owners and it is a condition of accessing publications that users recognise and abide by the legal requirements associated with these rights.

- Users may download and print one copy of any publication from the public portal for the purpose of private study or research.

- You may not further distribute the material or use it for any profit-making activity or commercial gain

- You may freely distribute the URL identifying the publication in the public portal.

If the publication is distributed under the terms of Article 25fa of the Dutch Copyright Act, indicated by the "Taverne" license above, please follow below link for the End User Agreement:

www.tue.nl/taverne

Take down policy

If you believe that this document breaches copyright please contact us at:

openaccess@tue.nl

providing details and we will investigate your claim. 


\section{Instituut Wiskundige Dienstverlening Eindhoven}

REPORT IWDE 90-06

THE SPECTRUM OF AN INHOMOGENEOUS

MEMBRANE

\section{J. Molenaar}

July 1990

Den Dolech 2

Postbus 513

$5600 \mathrm{MB}$ Eindhoven 


\title{
THE SPECTRUM OF AN INHOMOGENEOUS MEMBRANE
}

\author{
J. Molenaar
}

Report IWDE 90-06

July 1990

The author likes to thank Prof.dr. J. Boersma for critical reading of the manuscript and useful suggestions. 


\section{Introduction}

In this report we analyze the spectrum of a vibrating membrane with inhomogeneous density and search for a geometry with overtone frequencies which fit harmonically to a fundamental frequency. Then, the membrane will have a clear pitch, which makes it attractive for application in a certain class of musical instruments, namely the kettle drums. The present investigations are part of a study after the relationship between the performance of these instruments and their (many) geometrical parameters. Kettle drums consist of a circular membrane stretched over a kettle. The vibration modes of the system as a whole are the result of the interactions between the vibration modes of membrane, kettle and enclosed air. For an introduction into the physics of kettle drums we refer to [1]. In most kettle drums membranes of homogeneous density are used. The determination of the modes of such membranes (without kettle and in vacuo) may be considered as one of the standard problems in mathematical physics, which has been solved already long ago. The corresponding spectrum follows from the zeros of Bessel functions and is clearly inharmonic. In a certain Indian type of kettle drums, the so-called tabla, membranes of non-uniform density are applied. It is generally assumed that the presence of the inhomogeneities improves the harmonicity of the instrument. To focus on this latter idea, we study here a quite simple model in which the kettle and the air load are ignored.

We first show in $\$ 2$ how the spectrum of a stretched string of inhomogeneous density can be derived. Though this system is nearly trivial, it is quite appropriate to demonstrate the main ideas. The insights obtained this way are in $\$ 3$ carried over to the inhomogeneous membrane with circular symmetry. Thanks to the symmetry the theory involved strongly resembles the inhomogeneous string case. In $\$ 4$ the geometry without circular symmetry is dealt with. The lack of symmetry leads to substantial complications in determining the spectrum. The eigenfrequencies now follow from the zeros of the determinant of a matrix of infinite dimensions. In practice this matrix may appropriately be truncated if one is only interested in the lowest part of the spectrum. In $\$ 5$ numerical results for several geometries are presented. The Appendices A, B, C and D contain theoretical results, which are wider applicable than only within the context of the present case study. In references [2-8] results are published which have a close relationship with the results in $\$ \S 4$ and 5 . 


\section{The inhomogeneous string}

Consider an inhomogeneous string. The geometrical parameters are as indicated in Fig. 1, where the situation in rest is drawn.

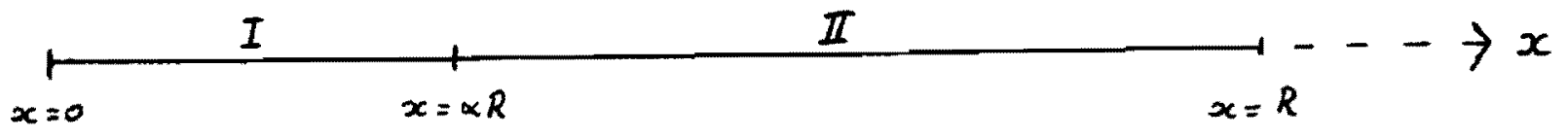

Figure 1.

The string is clamped at the end points $x=0$ and $x=R$. In region I, i.e. the interval $[0, \alpha R]$ with $0<\alpha<1$, the string has uniform density $\rho$; in region II it has uniform density $\delta \rho$ with multiplication factor $\delta>0$. In rest the stretched string has uniform tension $T$. The transversal displacement $u(t, x)$ satisfies the string equation

$$
\gamma^{2} u_{t t}=u_{x x}
$$

if $|u|$ and $\left|u_{x}\right|$ remain small with respect to $R$ and 1 respectively. In that case, $T$ can be considered to remain constant and uniform when the string vibrates. The function $\gamma^{2}$ takes two values, namely $\gamma^{2}=\gamma_{1}^{2}=\rho / T$ in region I and $\gamma^{2}=\gamma_{2}^{2}=\delta \rho / T$ in region II. The boundary conditions on $u$ are

$$
u(t, 0)=u(t, R)=0, t \geq 0
$$

and the initial conditions are given by

$$
\begin{array}{ll}
u(0, x)=u_{0}(x) & , 0 \leq x \leq R \\
u_{i}(0, x)=0 & , 0 \leq x \leq R .
\end{array}
$$

Equation (2.1) can be solved by separation of variables. We search for solutions of the general form $u(t, x)=f(t) g(x)$, which satisfy the homogeneous boundary conditions (2.2). Then, we obtain the ordinary differential equation (ODE) 


$$
f_{t}=\mu f
$$

for the time evolution of the system and the ODE

$$
g_{x x}=\gamma^{2} \mu g
$$

for the spatial distribution The separation constant $\mu$ is still to be determined from the boundary conditions. From (2.2) we find the conditions

$$
g(0)=g(R)=0
$$

These conditions can be satisfied only if $\mu<0$. For convenience we write $\mu=-\lambda^{2}$ with $\lambda \in \mathbb{R}$.

The allowed values for $\lambda$ form the frequency spectrum of the system. These values are found by solving the ODE (2.5) together with its boundary conditions (2.6). This is a regular SturmLiouville problem. See, e.g. [9-12]. The solution can in general be written as

$$
\begin{aligned}
g(x) & =A_{\lambda} \sin \left(\gamma_{1} \lambda x\right) & \text { in I } \\
& =B_{\lambda} \sin \left(\gamma_{2} \lambda x\right)+C_{\lambda} \cos \left(\gamma_{2} \lambda x\right) & \text { in II . }
\end{aligned}
$$

In writing (2.7) we already applied the condition $g(0)=0$. The condition $g(R)=0$ could be dealt with in the same way by writing $g(x)=B_{\lambda} \sin \left(\gamma_{2} \lambda(x-R)\right)$. We omit this reduction here to maintain the correspondence between $\S \S 2$ and 3 . The condition $g(R)=0$ and the continuity conditions on $g$ and $g_{x}$ at $x=R$ yield the linear equations

$$
\begin{aligned}
\sin \left(\gamma_{1} \lambda \alpha R\right) A_{\lambda} & =\sin \left(\gamma_{2} \lambda \alpha R\right) B_{\lambda}+\cos \left(\gamma_{2} \lambda \alpha R\right) C_{\lambda} \\
\gamma_{1} \cos \left(\gamma_{1} \lambda \alpha R\right) A_{\lambda} & =\gamma_{2} \cos \left(\gamma_{2} \lambda \alpha R\right) B_{\lambda}-\gamma_{2} \sin \left(\gamma_{2} \lambda \alpha R\right) C_{\lambda} \\
0 & =\sin \left(\gamma_{2} \lambda R\right) B_{\lambda}+\cos \left(\gamma_{2} \lambda R\right) C_{\lambda} .
\end{aligned}
$$

This system has a non-trivial solution if

$$
\operatorname{det} \mathbf{G}(\lambda)=0
$$

with the matrix $\mathbf{G}$ defined by

$$
\mathbf{G}(\lambda)=\left[\begin{array}{ccc}
-\sin \left(\gamma_{1} \lambda \alpha R\right) & \sin \left(\gamma_{2} \lambda \alpha R\right) & \cos \left(\gamma_{2} \lambda \alpha R\right) \\
-\gamma_{1} \cos \left(\gamma_{1} \lambda \alpha R\right) & \gamma_{2} \cos \left(\gamma_{2} \lambda \alpha R\right) & -\gamma_{2} \sin \left(\gamma_{2} \lambda \alpha R\right) \\
0 & \sin \left(\gamma_{2} \lambda R\right) & \cos \left(\gamma_{2} \lambda R\right)
\end{array}\right] .
$$

From (2.10) we obtain a discrete spectrum $\lambda_{m}, m=1,2,3, \cdots$, with $\lambda_{m}$ the $m$-th positive zero of det $\mathbf{G}$. Note, that in all of the special cases i) $\alpha=0, \gamma_{2}=\gamma$, ii) $\alpha=1, \gamma_{1}=\gamma$, and iii) $\gamma_{1}=\gamma_{2}=\gamma$, $\alpha \neq 0, \alpha \neq 1$, equation (2.10) with (2.11) reduces to the condition $\sin (\gamma \lambda R)=0$, so that the wellknown spectrum of the homogeneous string $\lambda_{n}=\frac{\pi m}{\gamma R}, m=1,2,3, \cdots$, is obtained.

Equations (2.9) determine the coefficients of $g$ in $(2.7,8)$ except for a constant factor. So, the 
eigenfunction $g_{m}$ of (2.5) with (2.6) corresponding to eigenvalue $\lambda_{m}$ may be written as

$$
\begin{aligned}
g_{m}(x) & =A_{m} \sin \left(\gamma_{1} \lambda_{m} x\right) & \text { in I } \\
& =B_{m} \sin \left(\gamma_{2} \lambda_{m} x\right)+C_{m} \cos \left(\gamma_{2} \lambda_{m} x\right) & \text { in II }
\end{aligned}
$$

in which the triple $\left(A_{m}, B_{m}, C_{m}\right)$ is derived from the triple $\left(A_{\lambda}, B_{\lambda}, C_{\lambda}\right)$ by normalizing $g_{m}$ with respect to the inner product

$$
(f, g)=\int_{0}^{R} \gamma^{2}(x) f(x) g(x) d x
$$

for functions $f, g$ which are square-integrable on $[0, R]$. Because the $g_{m}$ are the eigenfunctions corresponding to the non-degenerate eigenvalues $\lambda_{m}$ of the Sturm-Liouville problem (2.5) with (2.6), they are orthonormal, thus

$$
\left(g_{m}, g_{m^{\prime}}\right)=\delta_{m, m^{\prime}}
$$

and form a basis for the space $L_{2}([0, R])$ of square-integrable functions on $[0, R]$. For example, the starting profile $u_{0}(x)$ can be written as the Fourier series

$$
u_{0}(x)=\sum_{m=1}^{\infty}\left(u_{0}, g_{m}\right) g_{m}(x) .
$$

To each $\lambda_{m}$ a basis function $f_{m}(t) g_{m}(x)$ corresponds with $g_{m}$ given by (2.12). The function $f_{m}(t)$ satisfies (2.4) with $\mu=-\lambda_{m}^{2}$ and has the general form

$$
f_{m}(t)=D_{m} \cos \left(\lambda_{m} t\right)+E_{m} \sin \left(\lambda_{m} t\right) .
$$

Equation (2.1) is linear and homogeneous. Each linear combination of the basis functions also satisfies (2.1) and the homogeneous conditions (2.2). The properties of the $g_{m}$ assure, that the coefficients can be chosen such that initial conditions (2.3a,b) are satisfied. From (2.3b) it follows that all $E_{m}$ vanish; from (2.3a) we have $D_{m}=\left(u_{0}, g_{m}\right)$. The general solution of (2.1) with (2.2) and $(2.3 \mathrm{a}, \mathrm{b})$ is thus

$$
u(t, x)=\sum_{m=1}^{\infty}\left(u_{0}, g_{m}\right) g_{m}(x) \cos \lambda_{m} t
$$

If one chooses $u_{0}$ to coincide with a particular eigenfunction $g_{m}$, the vibration is periodic:

$$
u(t, x)=g_{m}(x) \cos \left(\lambda_{m} t\right)
$$




\section{The inhomogeneous membrane: circular symmetry}

We consider a circular membrane with a circular inhomogeneity in the density. The centres of membrane and inhomogeneity coincide as indicated in Fig. 2. The membrane radius is $R$, the inhomogeneity has radius $\alpha R, 0<\alpha<1$.

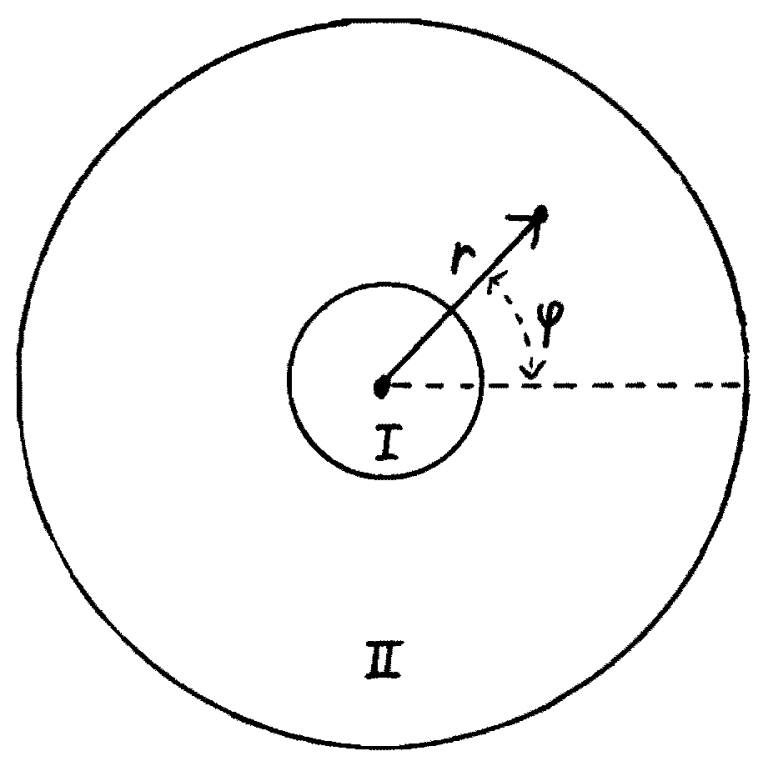

Figure 2.

The inhomogeneity is referred to as region I. It has homogeneous density $\delta \rho$ with multiplication factor $\delta>0$. The rest of the membrane is referred to as region II and has density $\rho$. We use polar coordinates $(r, \phi)$ as indicated in Fig. 2 . The transversal membrane displacement $u(t, r, \phi)$ satisfies the wave equation

$$
r^{2} u_{t t}=\Delta u=u_{r r}+\frac{1}{r} u_{r}+\frac{1}{r^{2}} u_{\phi \phi}
$$

with $\gamma^{2}=\gamma_{1}^{2}=\delta \rho / T$ in region I and $\gamma^{2}=\gamma_{2}^{2}=\rho / T$ in region II, with $T$ the tension. The linear equation (3.1) only holds if $|u|$ and $|\nabla u|$ remain small with respect to $R$ and 1 respectively. Then, the tension can be assumed to be constant in time and uniform over the membrane.

The initial conditions on the solutions of (3.1) are for $0 \leq r \leq R$

$$
\begin{aligned}
& u(0, r, \phi)=u_{0}(r, \phi) \\
& u_{i}(0, r, \phi)=0
\end{aligned}
$$


with $u_{0}$ a given starting profile. The boundary conditions with respect to $r$ are

$$
\begin{aligned}
& |u(t, 0, \phi)|<\infty, t \geq 0 \\
& u(t, R, \phi)=0, \quad t \geq 0 .
\end{aligned}
$$

With respect to $\phi$ we have the periodicity condition

$$
u(t, r, \phi)=u(t, r, \phi+2 \pi), t \geq 0,0 \leq r \leq R, \forall \phi .
$$

Just as for (2.1) the solutions of (3.1) can be obtained via separation of variables. We follow the same procedure as in $\$ 2$ and search for basis solutions of the form $u(t, r, \phi)=f(t) g(r, \phi)$. This leads to the equations

$$
f_{t}=\mu f
$$

and

$$
g_{r r}+\frac{1}{r} g_{r}+\frac{1}{r^{2}} g_{\phi \phi}=r^{2} \mu g .
$$

To solve (3.6), we again separate variables by assuming $g(r, \phi)=p(r) q(\phi)$. The resulting equation for $q$ is

$$
q_{\phi \phi}=-v q
$$

with periodicity condition $q(\phi)=q(\phi+2 \pi)$ and the equation for $p$ reads as

$$
r^{2} p_{r r}+r p_{r}+\left(-r^{2} \mu r^{2}-v\right) p=0
$$

with boundary conditions $|p(0)|<\infty$ and $p(R)=0$.

From the periodicity condition on $q$ we have $v=n^{2}, n \in \mathbb{N}$ (including 0 ). The eigenvalues are for $n \geq 1$ twofold degenerate. It is convenient to introduce a compact notation for the corresponding eigenfunctions. The eigenfunctions are labeled by the pair $L=(n, k)$ with $n \in \mathbb{N}$ and $k=0$ or 1. We define $c s_{0,0}=(2 \pi)^{-1 / 2}, c s_{0,1}=0$ and for $n \geq 1$

$$
\begin{aligned}
c s_{L}(\phi) & =\pi^{-1 / 2} \cos n \phi, \quad k=0 \\
& =\pi^{-1 / 2} \sin n \phi, \quad k=1 .
\end{aligned}
$$

The $c s_{L}$ form an orthonormal set of solutions of (3.8) with respect to the inner product

$$
(f, g)=\int_{0}^{2 \pi} f(\phi) g(\phi) d \phi=\delta_{L, L^{\prime}}
$$

for functions $f$ and $g$ which are square-integrable on $0 \leq \phi \leq 2 \pi$.

The orthonormality of $c s_{L}$ and $c s_{L^{\prime}}$ is easily established by integrating but follows also directly from the general theory on regular, periodic Sturm-Liouville problems. See [9-12]. The $c s_{L}$ form an orthonormal basis in $L_{2}([0,2 \pi])$, as is well known from Fourier series theory.

Equation (3.9) is Bessel's differential equation. The boundary conditions on $p$ are satisfied only if 
$\mu<0$, say $\mu=-\lambda^{2}, \lambda \in \mathbb{R}$. The two linearly independent solutions for fixed $\nu=n^{2}$ are $J_{n}(\gamma \lambda r)$ and $Y_{n}(\gamma \lambda r)$, the Bessel functions of the first and second kind respectively. Both solutions are admissible in region II, but in region I only $J_{n}$ may be used, because $Y_{n}$ is singular at $r=0$. From the solutions for $p$ and $q$ we may construct a general expression for the solution $g$ of (3.6). For given $\mu=-\lambda^{2}$ we have

$$
\begin{aligned}
& g(\mathbf{x})=\sum_{L} A_{L} J_{L}\left(\gamma_{1} \lambda \mathbf{x}\right) \quad \text { in } \mathrm{I} \\
& =\sum_{L}\left[B_{L} J_{L}\left(\gamma_{2} \lambda \mathbf{x}\right)+C_{L} Y_{L}\left(\gamma_{2} \lambda \mathbf{x}\right)\right] \text { in II , }
\end{aligned}
$$

where we use the shorthand notation

$$
J_{L}(\mathbf{x})=J_{n}(r) c s_{L}(\phi)
$$

with $\mathbf{x}=(r, \phi)$ in polar coordinates. For $Y_{L}(\mathbf{x})$ a similar definition as (3.14a) holds. In the following, we shall sometimes write out the argument of $J_{L}$ in polar coordinates

$$
J_{L}(\mathbf{x})=J_{L}(r, \phi) .
$$

The admissible $\lambda$-values, i.e. the frequency spectrum of the system, are to be determined from the boundary conditions on $g(r)$. They are for $0 \leq \phi<2 \pi$ :

i) $g$ is continuous at $r=\alpha R$

ii) $\partial g / \partial r$ is continuous at $r=\alpha R$

iii) $g(R)=0$.

Application of these conditions leads to three linear equations for the coefficients $A, B$ and $C$ in (3.12) and (3.13). In the first instance, these equations contain summations over $L$. However, by use of the orthonormality property (3.11) the equations can be shown to hold per $L$ value separately. Furthermore, the equations for fixed $n \geq 1$ and $k=0$ and 1 appear to be identical. The equations are

$$
\begin{aligned}
& J_{n}\left(\gamma_{1} \alpha R \lambda\right) A_{n}=J_{n}\left(\gamma_{2} \alpha R \lambda\right) B_{n} \quad+Y_{n}\left(\gamma_{2} \alpha R \lambda\right) C_{n} \\
& \gamma_{1} J_{n}{ }^{\prime}\left(\gamma_{1} \alpha R \lambda\right) A_{n}=\gamma_{2} J_{n}{ }^{\prime}\left(\gamma_{2} \alpha R \lambda\right) B_{n}+\gamma_{2} Y_{n}{ }^{\prime}\left(\gamma_{2} \alpha R \lambda\right) C_{n} \\
& 0=J_{n}\left(\gamma_{2} R \lambda\right) B_{n} \quad+Y_{n}\left(\gamma_{2} R \lambda\right) C_{n}
\end{aligned}
$$

with $J_{n}{ }^{\prime}$ and $Y_{n}{ }^{\prime}$ the derivatives of $J_{n}$ and $Y_{n}$ respectively, and $\left(A_{n}, B_{n}, C_{n}\right)=\left(A_{n 0}, B_{n 0}, C_{n 0}\right)=$ $\left(A_{n 1}, B_{n 1}, C_{n 1}\right)$. A non-trivial solution is found if for $n \in \mathbb{N}$

$$
\operatorname{det} \mathbf{G}_{n}(\lambda)=0
$$

with the matrix $\mathbf{G}_{n}$ given by 


$$
\mathrm{G}_{n}(\lambda)=\left(\begin{array}{ccc}
-J_{n}\left(\gamma_{1} \alpha R \lambda\right) & J_{n}\left(\gamma_{2} \alpha R \lambda\right) & Y_{n}\left(\gamma_{2} \alpha R \lambda\right) \\
-\gamma_{1} J_{n}^{\prime}\left(\gamma_{1} \alpha R \lambda\right) & \gamma_{2} J_{n}{ }^{\prime}\left(\gamma_{2} \alpha R \lambda\right) & \gamma_{2} Y_{n}{ }^{\prime}\left(\gamma_{2} \alpha R \lambda\right) \\
0 & J_{n}\left(\gamma_{2} R \lambda\right) & Y_{n}\left(\gamma_{2} R \lambda\right)
\end{array}\right]
$$

From (3.16) we obtain a discrete frequency spectrum $\left\{\lambda_{n m}\right)$ with $\lambda_{n m}$ the $m$-th positive zero of det $\mathbf{G}_{n}(\lambda)$. In the special case $\gamma_{1}=\gamma_{2}=\gamma$ the determinant of $\mathbf{G}_{n}$ is conveniently expanded according to the last row. Then, condition (3.16) reduces to

$$
\begin{aligned}
\operatorname{det} \mathbf{G}_{n}(\lambda) & =\gamma J_{n}(\gamma R \lambda)\left[J_{n}(\gamma \alpha R \lambda) Y_{n}{ }^{\prime}(\gamma \alpha R \lambda)-J_{n}{ }^{\prime}(\gamma \alpha R \lambda) Y_{n}(\gamma \alpha R \lambda)\right] \\
& =\frac{2}{\pi \alpha R \lambda} J_{n}(\gamma R \lambda) .
\end{aligned}
$$

Here, we use the general property that the Wronskian of $J_{n}(z)$ and $Y_{n}(z)$, which appears in (3.18) between the brackets, is equal to $2 / \pi z$. So, we indeed obtain the known result that the eigenfrequencies $\lambda_{n m}$ of a homogeneous, circular membrane are given by the zeros of the $J_{n}$. Note, that the same result is found by letting $\alpha \rightarrow 1$ in (3.17). However, one has to be careful in taking the limit $\alpha \rightarrow 0$, because expression (3.13) is no longer valid in that case.

We have seen, that for a fixed value of $\lambda_{n m}$ with $n \geq 1$ equation (3.6) for $g(r, \phi)$ has two solutions, which are labeled by $k=0$ and $k=1$. For $n=0$ we only have $k=0$. The complete set of solutions of (3.6) are denoted by $\left\{g_{n m k}\right\}$ with $n \in \mathbb{N}, m \in \mathbb{N}$ and $k=0$ or 1. Note, that these solutions are orthogonal by construction with respect to the inner product

$$
(f, g)=\int_{0}^{R} \int_{0}^{2 \pi} f(r, \phi) g(r, \phi) \gamma^{2}(r) r^{2} d r d \phi
$$

for functions $f, g$ which are square-integrable on the disc with radius $R$.

It is convenient to normalize them, after which it holds that

$$
\left(g_{n m k}, g_{n^{\prime} m^{\prime} k^{\prime}}\right)=\delta_{n, n^{\prime}} \delta_{m, m^{\prime}} \delta_{k, k^{\prime}}
$$

The solution of (3.1) is

$$
u(t, r, \phi)=\sum_{n=0}^{\infty} \sum_{m=1}^{\infty} \sum_{k=0}^{1}\left(u_{0}, g_{n m k}\right) g_{n m k}(r, \phi) \cos \left(\lambda_{n m} t\right) .
$$

If the initial displacement profile $u_{0}$ is equal to one of the eigenfunctions $g_{n m k}$ of the system, this profile will vibrate at eigenfrequency $\lambda_{n m}$. The eigenfunctions $g_{n m 0}$ and $g_{n m 1}$ are transformed into each other under a rotation over an angle $\pi / 2 n$. 


\section{The inhomogeneous membrane: no circular symmetry}

In this section we consider a circular membrane with a circular inhomogeneity, the centre of which does not coincide with that of the membrane. Then, no circular symmetry is present, which has far reaching consequences. The geometry is drawn in Fig. 3a, wherein also some symbols to be used in the following are indicated.

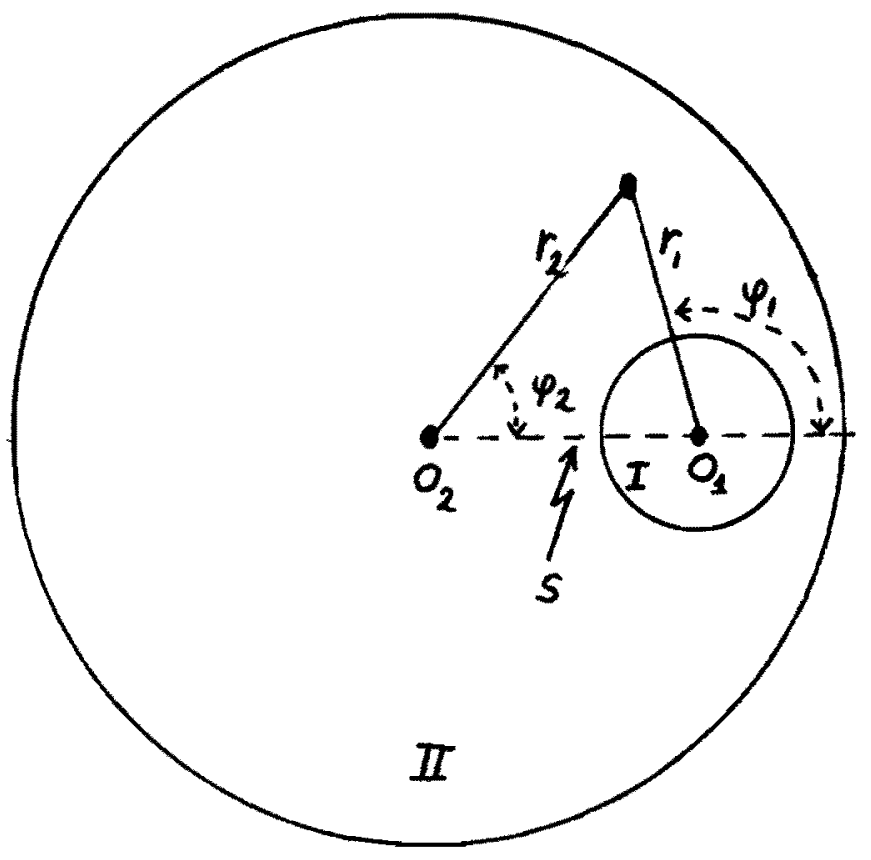

Fig. 3a.

The circular membrane has radius $R$, centre $\mathrm{O}_{2}$ and homogeneous density $\rho$ except for the circular inhomogeneity, which has homogeneous density $\delta \rho$ with multiplication factor $\delta>0$. The inhomogeneity is referred to as region I. It has radius $\alpha R$, with $0<\alpha<1$, and centre $O_{1}$. The vector $s=O_{2}-O_{1}$ connecting the centres has length $\beta R$ with $0 \leq \beta \leq(1-\alpha)$.

We assume the transversal displacement $u$ to be governed by (3.1). After separation of the time and the spatial variables the spatial part $g$ of the solution has to satisfy

$$
\gamma^{2} \lambda^{2} g=\Delta g
$$

with $\gamma^{2}=\gamma_{1}^{2}=\delta \rho / T, \delta>0$ in region I and $\gamma^{2}=\gamma_{2}^{2}=\rho / T$ in region II. The tension $T$ is assumed to be constant in time and uniform in space. In terms of the polar coordinates $\left(r_{1}, \phi_{1}\right)$ as indicated in Fig. 3 the solution of (4.1) can be quite generally written as 


$$
\begin{aligned}
g\left(r_{1}, \phi_{1}\right) & =\sum_{L} A_{L} J_{L}\left(\gamma_{1} \lambda r_{1}, \phi_{1}\right) \\
& =\sum_{L}\left(B_{L} J_{L}\left(\gamma_{2} \lambda r_{1}, \phi_{1}\right)+C_{L} Y_{L}\left(\gamma_{2} \lambda r_{1}, \phi_{1}\right)\right) \text { in II }
\end{aligned}
$$

with $J_{L}$ and $Y_{L}$ defined in (3.14). In this expression it is already taken into account that $\left|g\left(0, \phi_{1}\right)\right|<\infty$, so that the $Y_{L}$ do not appear in region I. The other conditions on $g$ are:

i) $g$ is continuous at $r_{1}=\alpha R$

ii) $\partial g / \partial r_{1}$ is continuous at $r_{1}=\alpha R$

iii) $g=0$ at $r_{2}=R$.

Conditions i) and ii) can be dealt with as in $\$ 3$. Multiplication by $c s_{L}\left(\phi_{1}\right)$ and integration over $\phi_{1}$ yields per $L$-value the equations

$$
\begin{aligned}
& J_{n}\left(\gamma_{1} \alpha R \lambda\right) A_{L}=J_{n}\left(\gamma_{2} \alpha R \lambda\right) B_{L}+Y_{n}\left(\gamma_{2} \alpha R \lambda\right) C_{L} \\
& \gamma_{1} J_{n}^{\prime}\left(\gamma_{1} \alpha R \lambda\right) A_{L}=\gamma_{2} J_{n}^{\prime}\left(\gamma_{2} \alpha R \lambda\right) B_{L}+\gamma_{2} Y_{n}^{\prime}\left(\gamma_{2} \alpha R \lambda\right) C_{L} .
\end{aligned}
$$

Note, that the equations for $k=0$ and $k=1$ are identical. This is not the case if we apply condition iii), because then the asymmetry of the system comes in. Application of condition iii) requires the expression of $g$ in terms of the polar coordinates $\left(r_{2}, \phi_{2}\right)$ instead of $\left(r_{1}, \phi_{1}\right)$ used in (4.2). To that end, we invoke the expansion rules

$$
\begin{aligned}
& J_{L}\left(\mathbf{x}_{1}\right)=\sum_{L^{\prime}} M_{L^{\prime}}(\mathbf{s}) J_{L^{\prime}}\left(\mathbf{x}_{2}\right) \\
& Y_{L}\left(\mathbf{x}_{1}\right)=\sum_{L^{\prime}} M_{L^{\prime}}(\mathbf{s}) Y_{L^{\prime}}\left(\mathbf{x}_{2}\right)
\end{aligned}
$$

which hold for vectors with $\mathbf{x}_{1}=\mathbf{s}+\mathbf{x}_{2}$.

Note, that the matrix $\mathbf{M}$ only depends on the shift of origins $\mathbf{s}=\mathrm{O}_{2}-\mathrm{O}_{1}$ of the coordinate systems under consideration as denoted in Fig. 3. Equations $(4,4 a, b)$ are derived in Appendices A-C (see formula (B.9)). In Appendix D alternative expressions are given cited from the literature. Expansion (4.4a) holds without geometrical restriction, whereas expansion (4.4b) is in this form only valid if $|\mathbf{s}|<\left|\mathbf{x}_{2}\right|$. If we substitute $(4.4 \mathrm{a}, \mathrm{b})$ into (4.2) and apply condition iii), we obtain the equation

$$
\sum_{L, L^{\prime}}\left[B_{L} M_{L L^{\prime}}\left(\gamma_{2} \lambda \mathbf{s}\right) J_{L^{\prime}}\left(\gamma_{2} \lambda R, \phi_{2}\right)+C_{L} M_{L L^{\prime}}\left(\gamma_{2} \lambda s\right) Y_{L^{\prime}}\left(\gamma_{2} \lambda R, \phi_{2}\right)\right]=0
$$

One of the summations disappears if this equation is multiplied by some $c s_{L}\left(\phi_{2}\right)$ and integrated over $\phi_{2}$. After some relabeling we obtain per $L=(n, k)$ value the equation

$$
\sum_{L^{\prime}}\left[J_{n}\left(\gamma_{2} \lambda R\right) M_{L L^{\prime}}^{T}\left(\gamma_{2} \lambda \mathbf{s}\right) B_{L^{\prime}}+Y_{n}\left(\gamma_{2} \lambda R\right) M_{L L^{\prime}}^{T}\left(\gamma_{2} \lambda s\right) C_{L^{\prime}}\right]=0
$$

Note that the equations for $k=0$ and $k=1$ are not identical. This degeneracy, which was present in the system in $\$ 3$, is broken up here. Another consequence of the lack of symmetry is the 
coupling between coefficients with different $n$ values, due to the summation in (4.6). However, it is important to realize that in (4.6) no coupling occurs between terms with $k^{\prime}=0$ and terms with $k^{\prime}=1$. In the polar coordinates $\left(r_{1}, \phi_{1}\right)$ in region I the vector $s$ has the representation $(|s|, 0)$. This implies that, because of property (C.3), $M_{L^{\prime}} \cdot\left(\gamma_{2} \lambda s\right)=0$ if $k \neq k^{\prime}$.

If we combine (4.3) and (4.6), a non-trivial solution for the coefficients exists if one of the following conditions hold

$$
\begin{aligned}
\operatorname{det} \mathbf{G}^{0}(\lambda) & =0 \\
\operatorname{det} \mathbf{G}^{1}(\lambda) & =0
\end{aligned}
$$

with $\mathbf{G}^{0}$ and $\mathbf{G}^{1}$ matrices of infinite dimensions. $\mathbf{G}^{0}$ corresponds with the equations with $k=0$ and $\mathrm{G}^{1}$ with the equations with $k=1$. These matrices are built up out of $3 \times 3$ blocks, which we label as $\mathbf{G}_{n, n^{\prime}}^{0}$ and $\mathbf{G}_{n, n^{\prime}}^{1}$. The diagonal blocks are given by

$$
\mathbf{G}_{n, n}^{0}=\left[\begin{array}{ccc}
-J_{n}\left(\gamma_{1} \alpha R \lambda\right) & J_{n}\left(\gamma_{2} \alpha R \lambda\right) & Y_{n}\left(\gamma_{2} \alpha R \lambda\right) \\
-\gamma_{1} J_{n}^{\prime}\left(\gamma_{1} \alpha R \lambda\right) & \gamma_{2} J_{n}^{\prime}\left(\gamma_{2} \alpha R \lambda\right) & \gamma_{2} Y_{n}^{\prime}\left(\gamma_{2} \alpha R \lambda\right) \\
0 & J_{n}\left(\gamma_{2} \lambda R\right) M_{L L}^{T}\left(\gamma_{2} \lambda s\right) & Y_{n}\left(\gamma_{2} \lambda R\right) M_{L L}^{T}\left(\gamma_{2} \lambda s\right)
\end{array}\right]
$$

with $L=(n, 0) . \mathbf{G}^{1}$ have a similar form with $L=(n, 1)$. The off-diagonal blocks are given by

$$
\mathbf{G}_{n, n^{\prime}}^{0}=\left[\begin{array}{lll}
0 & 0 & 0 \\
0 & 0 & 0 \\
0 & J_{n}\left(\gamma_{2} \lambda R\right) M_{L L^{\prime}}^{T}\left(\gamma_{2} \lambda s\right) & Y_{n}\left(\gamma_{2} \lambda R\right) M_{L L^{\prime}}^{T}\left(\gamma_{2} \lambda s\right)
\end{array}\right]
$$

with $L=(n, 0), L^{\prime}=\left(n^{\prime}, 0\right), \mathbf{G}_{n, n^{\prime}}^{1}$ has a similar form with $L=(n, 1)$ and $L^{\prime}=\left(n^{\prime}, 1\right)$. In the special case $s=0$ conditions $(4.7 \mathrm{a}, \mathrm{b})$ indeed reduce to condition (3.16) in view of property (B.11) of the shift matrix $\mathbf{M}$.

The spectrum of the system is in principle obtained from (4.7) with (4.8). In practice one has to cut off the $\mathbf{G}^{0}$ and $\mathbf{G}^{1}$ matrices in order to be able to calculate the determinants. Reliable values for this cut-off value must be determined numerically and will, of course, depend on the accuracy required. 


\section{Numerical results}

We have implemented conditions (4.7a) and (4.7b) with (4.8a) and (4.8b) numerically and calculated the spectra of several geometries. In all examples we fixed the radius, tension and density of the membrane at the values $R=1, T=1$ and $\rho=1$, respectively. The parameters $\alpha, \beta$ and $\delta$ (see Fig. 3) are varied successively. In all cases a cut-off value of $n=5$ for the matrices in $(4.8 \mathrm{a}, \mathrm{b})$ is used. We present results for four cases. In Figures $4 a, 5 a, 6 a, 7 a$ the corresponding spectra are given. In Figures $4 \mathrm{~b}, 5 \mathrm{~b}, 6 \mathrm{~b}, 7 \mathrm{~b}$ the same spectra are drawn with all eigenvalues divided by the frequency of the $(1,1)$ mode. The $(1,1)$ frequency is in general the fundamental one of a kettle drum, because the $(0,1)$ mode, which has a lower frequency, damps out much faster. A musical instrument conveys a clear sense of pitch if it has several harmonic overtones, the frequencies of which are related to the fundamental in whole-number ratios. From the latter figures one can find whether the presence of an inhomogeneity makes the membrane more or less "harmonic".

$-\quad 0 \leq \alpha \leq 1, \beta=0, \delta=2$.

See Fig. 4 a,b. This geometry has circular symmetry and the theory of $\$ 3$ applies. For $\alpha=0$ and $\alpha=1$ the membrane is homogeneous. In these limiting cases the spectrum is given, according to (3.18), by the zeros of the functions $J_{n}(\lambda)$ and $J_{n}(\sqrt{2} \lambda)$ respectively. The spectrum is given in Fig. 4a,b as a function of $\alpha$. For $n=0$ the eigenvalues are non-degenerate; for $n \geq 1$ they are two-fold degenerate. It is seen, that for a small, centrally positioned inhomogeneity $(\alpha<1 / 2)$, the eigenvalues with $n=0$ are most influenced. For $1 / 2<\alpha \leq 1$ all eigenvalues behave in nearly the same way. Going from $\alpha=0$ to $\alpha=1 / 2$ the total mass of the membrane is increased by a factor of $5 / 4$.

$-\alpha=0.5,0 \leq \beta \leq 0.5, \delta=2$.

See Fig. 5a,b. In this case the inhomogeneity is shifted away from the central position. For $\beta>0$ the circular symmetry is disturbed, so that the two-fold degeneracy of the eigenvalues with $n \geq 1$ as present in Fig. 4 is absent here. The $\beta=0$ values in Fig. $5 \mathrm{a}, \mathrm{b}$ correspond to the $\alpha=0.5$ values in Fig. $4 \mathrm{a}, \mathrm{b}$.

$-\quad 0 \leq \alpha \leq 0.5, \beta=0.5, \delta=2$.

See Fig. 6a,b. Here, the inhomogeneity is positioned decentrally and its radius is varied. For $\alpha=0$ the membrane is homogeneous and the degeneracies are present. Increasing $\alpha$ leads to a small decrease of the eigenvalues because the average density is enhanced. The $(1,1)$ and $(1,2)$ eigenvalues are mostly affected, especially in the $\alpha>0.25$ region.

$-\alpha=0.5, \beta=0.5,1 \leq \delta \leq 4$.

See Fig. $7 \mathrm{a}, \mathrm{b}$. In this case the influence of the density of an asymmetrically placed inhomogeneity of fixed size is studied. For $\delta=1$ we have a homogeneous membrane. Going from $\delta=1$ to $\delta=4$ the average density of the membrane is enhanced by a factor of $7 / 4$. As expected, most of the 
eigenvalues decrease for increasing $\delta$. This happens in quite a regular way except for the (4.1) and the $(2,2)$ frequencies which show an increasing behaviour for $\delta<1.5$.

In the geometries shown only one favourable situation is found, namely in Fig. $6 \mathrm{~b}$ with $\alpha=0.25$ á 0.30 . Then, the $(1,1),(0,2),(1,2),(2,2),(0,3),(5,1)$ frequencies are present in nearly the ratio's $1: 1.5: 2: 2.5$, the $(2,2),(0,3)$ and $(5,1)$ frequencies coinciding. In this case the fundamental frequency $(1,1)$ is considerably splitted up. This has little influence on the harmonicity, because one can filter out one of the two by striking the membrane at an appropriate non-central position. The $(0,2)$ mode, however, damps out relatively rapidly.

Our general conclusion is negative. In the usual kettledrum with homogeneous membrane the $(1,1),(2,1),(3,1)$, and $(4,1)$ frequencies form a harmonic series. For a membrane in vacuo we have not found a geometry in which these frequencies show a tendency to approach a harmonic series due to the introduction of an inhomogeneity. It is not clear whether the results for a membrane in vacuo may also be drawn with respect to the system consisting of membrane, kettle and air. If this is so we conclude that the inhomogeneities in the membranes of indian tablas play a role only in the fine tuning of these instruments and are not essential for the harmonicity of their spectra. 


\section{Appendix A}

In Appendices A, B and C we explicitly derive equations (4.4a,b) and an expression for the matrix $\mathbf{M}$. In Appendix $D$ it is shown that the form of $\mathbf{M}$ also follows from addition theorems known in the literature.

Let us consider the eigenvalue problem for the Laplace operator in two dimensions

$$
\Delta=\partial^{2} / \partial x^{2}+\partial^{2} / \partial y^{2}
$$

in Cartesian coordinates $(x, y)$ and

$$
\Delta=\partial^{2} / \partial r^{2}+(1 / r) \partial / \partial r+\left(1 / r^{2}\right) \partial^{2} / \partial \phi^{2}
$$

in polar coordinates $(r, \phi)$. So we study the equation

$$
-\Delta f=E f
$$

with boundary conditions

$$
\begin{aligned}
& |f(0)|<\infty \\
& f(\infty)=0 .
\end{aligned}
$$

The three-dimensional analogues of the results in Appendices A, B and C can be found in [13]. The minus sign in (A.3) is for convenience. Equation (A.3) can be interpreted in many ways. E.g., it can be read as the Schrödinger equation of a free particle with unit mass and energy $E$. The solutions of (A.3) can be obtained via separation of variables as shown in $\$ \$ 3$ and 4 . The eigenvalue spectrum is continuous with $0<E<\infty$. Each eigenvalue is infinitely times degenerate. We label the linearly independent eigenfunctions by the indices, also called quantumnumbers, $(E, n, k)$ with $n \in \mathbb{N}$ and $k=0$ or 1 . For convenience, we write $L=(n, k)$ and denote the eigenfunctions as $|E, L\rangle$ using Dirac notation. The $|E, L\rangle$ form an orthonormal basis in $L_{2}\left(\mathbb{R}^{2}\right)$ if we choose them as

$$
<\mathbf{x} \mid E, L>=\frac{1}{\sqrt{2}} J_{L}(\sqrt{E} \mathbf{x})=\frac{1}{\sqrt{2}} J_{n}(\sqrt{E} x) c s_{L}(\hat{x})
$$

with $c s_{L}$ defined by $(3.10)$ and $\mathbf{x}=(x, \hat{x})$ in polar coordinates. The orthonormality means that

$$
\left\langle E, L \mid E^{\prime}, L^{\prime}\right\rangle=\int_{\mathbb{R}^{2}}\langle E, L \mid \mathbf{x}\rangle\left\langle\mathbf{x} \mid E^{\prime}, L^{\prime}\right\rangle d \mathbf{x}=\delta\left(E-E^{\prime}\right) \delta_{L L^{\prime}}
$$

In (A.5) $\mathrm{x}$ is represented as $(x, \hat{x})$ with respect to a specific coordinate system. However, the Laplace operator is translation invariant and if we evaluate (A.3) with (A.4) with respect to a shifted origin, we obtain alternative eigenfunctions. We denote them by $\mid E, L ; \mathrm{s}>$ with $\mathrm{s}$ the origin shift. Note, that we only shift the origin but do not change the $\hat{x}=0$ direction, i.e. no rotation is applied. The $|E, L ; \mathbf{s}\rangle$ can be obtained from the $|E, L\rangle$ via the orthogonal shift operator: 


$$
|E, L ; \mathbf{s}>=\exp (\mathbf{s} \cdot \nabla)| E, L>
$$

with

$$
\nabla=\left[\begin{array}{l}
\partial / \partial x \\
\partial / \partial y
\end{array}\right)
$$

in Cartesian coordinates.

We note that the $\mid E, L ; \mathbf{s}>$ form an orthonormal basis for $L_{2}\left(\mathbb{R}^{2}\right)$ for all $\mathbf{s}$. This can be expressed as

$$
\sum_{L} \int_{0}^{\infty} d E|E, L ; \mathbf{s}\rangle\langle E, L ; \mathbf{s}|=\mathbf{1}
$$

with 1 the unity operator. The functions $|E, L ; \mathbf{s}\rangle$ and $\left|E^{\prime}, L^{\prime} ; \mathbf{s}^{\prime}\right\rangle$ are not mutually orthogonal if $\mathbf{s} \neq \mathbf{s}^{\prime}$. We define a matrix $\overline{\mathbf{M}}$ by

$$
\left\langle E, L ; \mathbf{s} \mid E^{\prime}, L^{\prime} ; \mathbf{s}^{\prime}\right\rangle=\bar{M}_{L L^{\prime}}\left(E, \mathbf{s}, \mathbf{s}^{\prime}\right) \delta\left(E-E^{\prime}\right) .
$$

We may explicitly write a delta function with respect to the energies here, because the shift operator $\nabla$ commutes with the Laplacian $\Delta=\nabla \cdot \nabla$. An explicit formula for $\bar{M}$ is derived in Appendix B. There it is shown, that $\overline{\mathbf{M}}$ does not depend on three arguments separately, but on only one (vector) argument. We therefore introduce

$$
M_{L L} \cdot\left(\sqrt{E}\left(\mathbf{s}-\mathbf{s}^{\prime}\right)\right)=\bar{M}_{L L} \cdot\left(E, \mathbf{s}, \mathbf{s}^{\prime}\right) .
$$

From property (A.9) and definition (A.10) we find for arbitrary vectors $s$ and $s^{\prime}$

$$
\sum_{L^{\prime \prime}} M_{L L^{\prime \prime}}(\mathbf{s}) M_{L^{\prime \prime} L^{\prime}}\left(\mathbf{s}^{\prime}\right)=M_{L L^{\prime}}\left(\mathbf{s}+\mathbf{s}^{\prime}\right) \text {. }
$$

If we take $L^{\prime}=(0,0)$ and use property (B.12), equation (A.12) reduces to

$$
J_{L}\left(\mathrm{~s}+\mathrm{s}^{\prime}\right)=\sum_{L^{\prime}} M_{L^{\prime}} \cdot(\mathrm{s}) J_{L^{\prime}}\left(\mathrm{s}^{\prime}\right) .
$$

Equation (4.4a) is thus a special case of (A.12). We derive equation (4.4b) from (4.4a) via formula (1) in $\$ 13.53$ of [14]. In the latter formula we choose $m=0$ and substitute the property

$$
Y_{n}(-x)=(-1)^{n} Y_{n}(x)+2 i(-1)^{n} J_{n}(x) .
$$

Then we obtain the definite integral

$$
\int_{0}^{\infty} \frac{x^{\rho-1}}{x^{2}-r^{2}} J_{n}(a x) J_{n^{\prime}}(b x) d x=\frac{1}{2} \pi i r^{\rho-2} J_{n}(a x) H_{n}^{(1)}(b x),
$$

in which $a$ and $b$ are real numbers with $b>a>0, r$ is a complex number with a positive imaginary part and $\rho$ is a positive integer with $\rho<4$. The Hankel function of the first kind $H^{(1)}$ is defined by $H_{n}^{(1)}(x)=J_{n}(x)+i Y_{n}(x)$. Equation (A.15) holds under the condition that $\rho+n+n^{\prime}$ is 
even. A special case is obtained by taking $n=0$ and $a=0$ :

$$
\int_{0}^{\infty} \frac{x^{p-1}}{x^{2}-r^{2}} J_{n}(b x) d x=\frac{1}{2} \pi i r^{\rho-2} H_{n}^{(1)}(b x)
$$

under the condition that $\rho+n$ is even.

If we substitute expression (B.9) for $\mathbf{M}$ into (A.13) and multiply all vectors involved by an arbitrary real number $x \geq 0$, we obtain the equation

$$
J_{L}\left(x\left(\mathbf{s}+\mathbf{s}^{\prime}\right)\right)=\sum_{L^{\prime}, L^{\prime \prime}} i^{\left(n-n^{\prime}+n^{\prime \prime}\right)} c_{L L^{\prime} L^{\prime \prime}} J_{L^{\prime \prime}}(x \mathbf{s}) J_{L^{\prime}}\left(x \mathbf{s}^{\prime}\right)
$$

From the properties of the Gaunt coefficient $c$ (see Appendix C) we have that $n+n^{\prime}+n^{\prime \prime}$ in the r.h.s. of (A.17) is even. So $n$ and $n^{\prime}+n^{\prime \prime}$ are both even or both odd. If we multiply both sides of (A.17) by the factor $x^{\rho-1} /\left(x^{2}-r^{2}\right)$ and integrate over $0 \leq x<\infty$, (A.15) is applicable to the r.h.s. and (A.16) to the 1.h.s. of (A.17) provided that $\rho+n^{\prime}+n^{\prime \prime}$ and thus $\rho+n$ are even. This requirement can be fulfilled because of the freedom in choice we have for $\rho$. The resulting equation is

$$
H_{L^{1}}^{(1)}\left(r\left(\mathrm{~s}+\mathrm{s}^{\prime}\right)\right)=\sum_{L^{\prime}} M_{L L^{\prime}}(r \mathrm{~s}) H_{L^{\prime}}^{(1)}\left(r \mathbf{s}^{\prime}\right)
$$

under the condition $\left|s^{\prime}\right|>|s|$. We may take the imaginary part of $r$ arbitrarily small, which implies that (A.18) also holds for $r=1$. The real part of (A.17) is in that case identical to (A.13) and (4.4a), and the imaginary part is just expansion (4.4b). 


\section{Appendix B}

To derive an explicit expression for the matrix $M$ in $(4.4 \mathrm{a}, \mathrm{b})$ we start from the equation (cf. (A.8) and (A.10))

$$
M_{L L^{\prime}}(\sqrt{E} \mathbf{s})=\left\langle E, L|\exp (\mathbf{s} \cdot \nabla)| E, L^{\prime}\right\rangle .
$$

The r.h.s. of (B.1) is most easily evaluated by the use of the eigenfunctions $\mid \mathbf{k}>$ of the operator $\nabla$. We have

$$
\nabla|\mathbf{k}>=\mathbf{k}| \mathbf{k}>
$$

with $\mid \mathrm{k}>$ a normalized plane wave in two dimensions

$$
\langle\mathbf{x} \mid \mathbf{k}\rangle=(2 \pi)^{-1} \exp (i \mathbf{k} \cdot \mathbf{x})
$$

and $\left\langle\mathbf{k} \mid \mathbf{k}^{\prime}\right\rangle=\delta\left(\mathbf{k}-\mathbf{k}^{\prime}\right)$. We insert the unity operator

$$
\int_{\mathbb{R}^{2}} d \mathbf{k}|\mathbf{k}><\mathbf{k}|=1
$$

into (B.1) and obtain for the r.h.s. the integral

$$
\int d \mathbf{k}\left\langle E, L|\mathbf{k}>\exp (i \mathbf{s} \cdot \mathbf{k})<\mathbf{k}| E, L^{\prime}\right\rangle \text {. }
$$

To evaluate the matrix element $\langle E, L \mid \mathbf{k}\rangle$ we expand $\langle\mathbf{x} \mid \mathbf{k}\rangle$ as follows, using (A.6):

$$
\begin{aligned}
\langle\mathbf{x} \mid \mathbf{k}\rangle & =\int d E \sum_{L}\langle\mathbf{x} \mid E, L\rangle\langle E, L \mid \mathbf{k}\rangle \\
& =\frac{1}{\sqrt{2}} \int d E \sum_{L} J_{n}(\sqrt{E} x) c s_{L}(\hat{x})\langle E, L \mid \mathbf{k}\rangle .
\end{aligned}
$$

If we compare this with the well-known expansion for a plane wave in two dimensions

$$
\exp (i \mathbf{k} \cdot \mathbf{x})=2 \pi \sum_{L} i^{n} J_{n}(k x) c s_{L}(\hat{x}) c s_{L}(\hat{k})
$$

and realize that $E=k^{2}$, we find

$$
\langle E, L \mid \mathbf{k}\rangle=\sqrt{2} i^{n} c s_{L}(\hat{k}) \delta\left(E-k^{2}\right) .
$$

By means of equations (B.7) and (B.8), expression (B.5) is easily evaluated, giving

$$
M_{L L^{\prime}}(\mathbf{s})=2 \pi \sum_{L^{\prime \prime}} i^{n-n^{\prime}+n^{\prime \prime}} c_{L L^{\prime} L^{\prime \prime}} J_{L^{\prime \prime}}(\mathbf{s})
$$

with the Gaunt coefficient $c_{L L^{\prime} L^{\prime \prime}}$ defined by

$$
c_{L L^{\prime} L^{\prime \prime}}=\int_{0}^{2 \pi} d \phi c s_{L}(\phi) c s_{L^{\prime}}(\phi) c s_{L^{\prime \prime}}(\phi) .
$$

In Appendix $C$ explicit expressions for $c_{L^{\prime} L^{\prime \prime}}$ are given. For $\mathrm{s}=0$ only the $L^{\prime \prime}=(0,0)$ term in the summation in (B.9) remains and we find 


$$
M_{L L^{\prime}}(\mathbf{0})=\delta_{L L^{\prime}} .
$$

If we take $L^{\prime}=(0,0)$ in (B.9), we obtain the property

$$
M_{L(0,0)}(\mathrm{s})=J_{L}(\mathrm{~s}) .
$$




\section{Appendix C}

Here, we present explicit expressions for the Gaunt coefficients

$$
c_{L L^{\prime} L^{\prime \prime}}=\int_{0}^{2 \pi} c s_{L}(\phi) c s_{L^{\prime}}(\phi) c s_{L^{\prime \prime}}(\phi) d \phi
$$

with $c s$ defined by (3.10). One can work out the product of sines and cosines using the identities

$$
\begin{aligned}
& \cos \phi \cos \phi^{\prime}=\frac{1}{2}\left(\cos \left(\phi+\phi^{\prime}\right)+\cos \left(\phi-\phi^{\prime}\right)\right) \\
& \sin \phi \cos \phi^{\prime}=\frac{1}{2}\left(\sin \left(\phi+\phi^{\prime}\right)+\sin \left(\phi-\phi^{\prime}\right)\right) \\
& \sin \phi \sin \phi^{\prime}=-\frac{1}{2}\left(\cos \left(\phi+\phi^{\prime}\right)-\cos \left(\phi-\phi^{\prime}\right)\right)
\end{aligned}
$$

This eventually yields (with $L=(n, k), L^{\prime}=\left(n^{\prime}, k^{\prime}\right)$ and $L^{\prime \prime}=\left(n^{\prime \prime}, k^{\prime \prime}\right)$ ):

$$
\begin{array}{rlrl}
c_{L L^{\prime} L^{\prime \prime}} & =0 & & \text { if } k+k^{\prime}+k^{\prime \prime} \text { is uneven } \\
& =\frac{1}{2}\left(\delta_{n, n^{\prime}+n^{\prime \prime}}+\delta_{n,\left|n^{\prime}-n^{\prime \prime}\right|}\right) & & \text { if } k=k^{\prime}=k^{\prime \prime}=0 \\
& =\frac{1}{2}\left(\delta_{n,\left|n^{\prime}-n^{\prime \prime}\right|}-\delta_{n, n^{\prime}+n^{\prime \prime}}\right) & & \text { if }\left(k, k^{\prime}, k^{\prime \prime}\right)=(0,1,1) \\
& =\frac{1}{2}\left(\delta_{n^{\prime},\left|n^{\prime \prime}-n\right|}-\delta_{n^{\prime}, n^{\prime \prime}+n}\right) & & \text { if }\left(k, k^{\prime}, k^{\prime \prime}\right)=(1,0,1) \\
& =\frac{1}{2}\left(\delta_{n^{\prime \prime},\left|n-n^{\prime}\right|}-\delta_{n^{\prime \prime}, n+n^{\prime}}\right) & \text { if }\left(k, k^{\prime}, k^{\prime \prime}\right)=(1,1,0) .
\end{array}
$$




\section{Appendix D}

Equations $(4.4 \mathrm{a}, \mathrm{b})$ and expression (B.9) also follow from an addition theorem derived by Graf, which is given in [14], section 11.3. In this appendix we partly adopt the notation of [14]. See Fig. 3b, in which the angle $\phi_{1}$ is defined otherwise than in Fig. 3a.

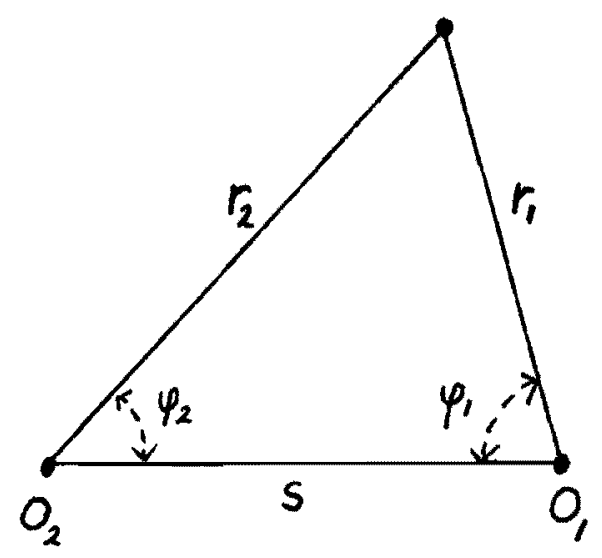

Fig. 3b.

From (2) and (3) in [14] we obtain the relation

$$
\exp \left( \pm n i\left(\pi-\phi_{1}-\phi_{2}\right) J_{n}\left(r_{1}\right)=\sum_{m=-\infty}^{+\infty} J_{n+m}\left(r_{2}\right) J_{m}(s) \exp \left( \pm m i \phi_{2}\right)\right.
$$

If we multiply both sides by $\exp \left( \pm n i \phi_{2}\right)$ and redefine the summation index we find

$$
(-1)^{n} J_{n}\left(r_{1}\right) \exp \left(\mp n i \phi_{1}\right)=\sum_{m=-\infty}^{+\infty} J_{m-n}(s) J_{m}\left(r_{2}\right) \exp \left( \pm m i \phi_{2}\right) \text {. }
$$

This leads to

$$
J_{n}\left(r_{1}\right) \cos \left(n \phi_{1}\right)=J_{n}(s) J_{0}\left(r_{2}\right)+\sum_{m=1}^{\infty}\left[(-1)^{n} J_{m-n}(s)+J_{m+n}(s)\right] J_{m}\left(r_{2}\right) \cos \left(m \phi_{2}\right)(\mathrm{D} .3)
$$

and

$$
J_{n}\left(r_{1}\right) \sin \left(n \phi_{1}\right)=\sum_{m=1}^{\infty}\left[(-1)^{n+1} J_{m-n}(s)+J_{m+n}(s)\right] J_{m}\left(r_{2}\right) \sin \left(m \phi_{2}\right) .
$$

For $Y_{n}$ similar relations hold. One clearly recognizes in (D.3) and (D.4) the same structure as in (4.4a,b). From (D.3) and (D.4) an expression for the transformation matrix $\mathbf{M}$ follows, which is identical to expression (B.9), provided that the difference in the definitions of $\phi_{1}$ in Figures $3 a$ and $3 \mathrm{~b}$ is taken into account. 


\section{References}

1. Rossing, T.D., The Physics of Kettledrums, Scientific American, November 1982, pp. $172-$ 178.

2. Nagaya K. and Hai Y., Free Vibration of Composite Membranes with Arbitrary Shape, J. Sound and Vibration (1985), 100 (1), pp. 123-134.

3. Nagaya K., Vibration of a Membrane having a Circular Outer Boundary and an Eccentric Circular Inner Boundary, J. Sound and Vibration (1977), 50 (4), pp. 545-551.

4. Nagaya K., Vibrations and Dynamic Response of Membranes with Arbitrary Shape, J. Applied Mech. (1978), 45, pp. 153-158.

5. De S., Vibrations of a Composite Annular Membrane, J. Sound and Vibration, 17 (1), pp. 13-23.

6. Leal C. and Sanchez-Hubert J., Perturbation of the Eigenvalues of a Membrane with a Concentrated Mass, Quarterly of Appl. Mech., Vol. XIVII (1), March (1989), pp. 93-103.

7. Hine H.J., Eigenvalues for a Uniform Fluid Waveguide with an Eccentric-Annulus CrossSection, J. Sound and Vibration (1971), 15 (3), pp. 295-305.

8. Kac M., Can One Hear the Shape of a Drum? Am. Math. Monthly (1966), 3, pp. 1-23.

9. Marchenko V.A., Sturm-Liouville Operators and Applications, Operator Theory; Advances and Applications, Vol. 22, Birkhäuser Verlag, Basel-Boston-Stuttgart, 1986.

10. Myint-U T. and Debnath L., Partial Differential Equations for Scientists and Engineers, North-Holland, New York, Amsterdam, London, 1987.

11. Courant R. and Hilbert D., Methods of Mathematical Physics, Vol. 1, Interscience Publishers, New York, 1953.

12. Ince E.L., Ordinary Differential Equations, (orig. ed. Longmans, Green, New York, 1926), Dover, New York, 1956.

13. Molenaar J., Multiple-scattering Theory Beyond the Muffin-Tin Approximation, J. Phys. C: Solid State Phys. 21 (1988), pp. 1455-1468.

14. Watson G.N., Theory of Bessel Functions, Cambridge University Press, Cambridge, 1966. 


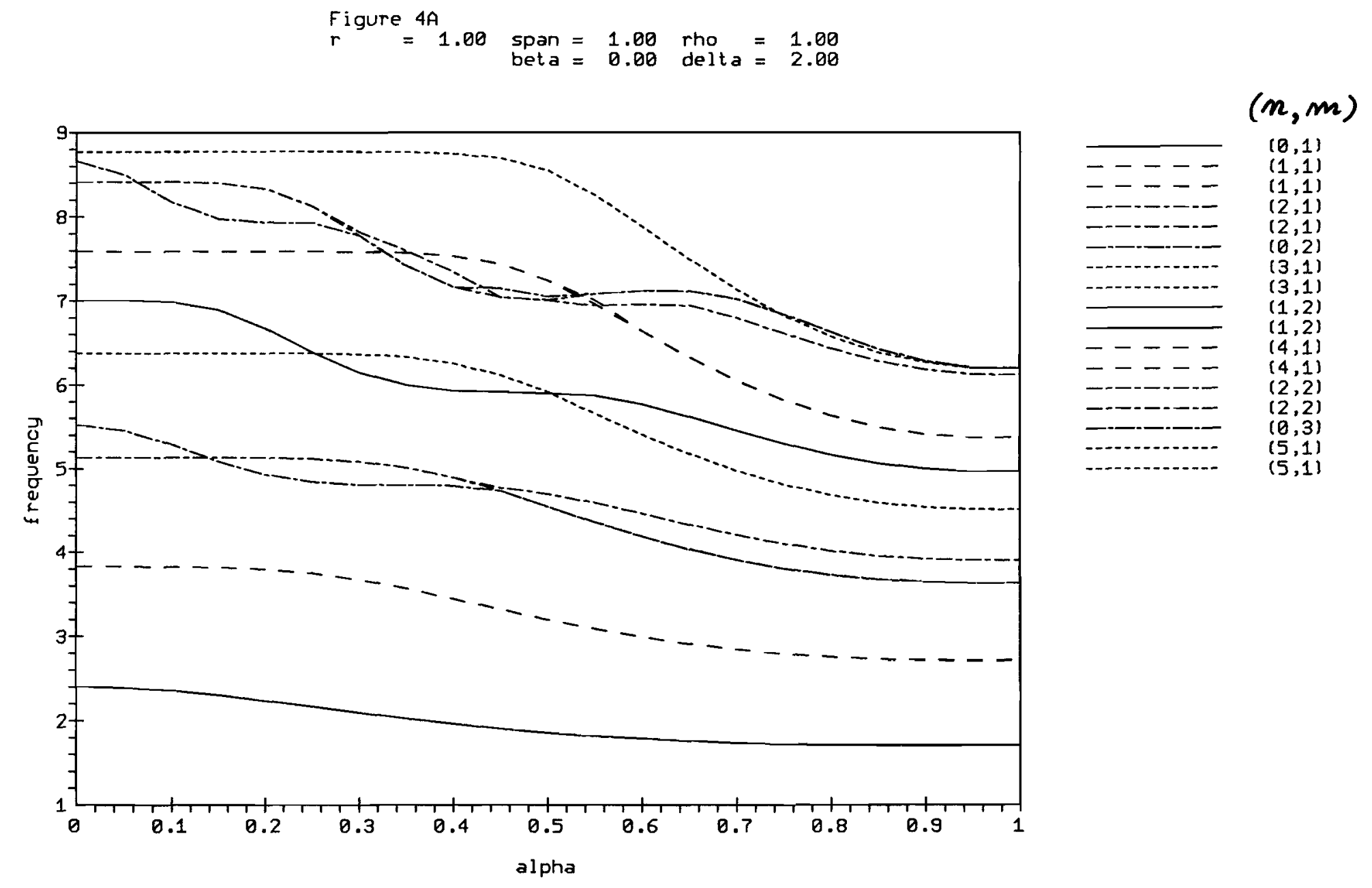




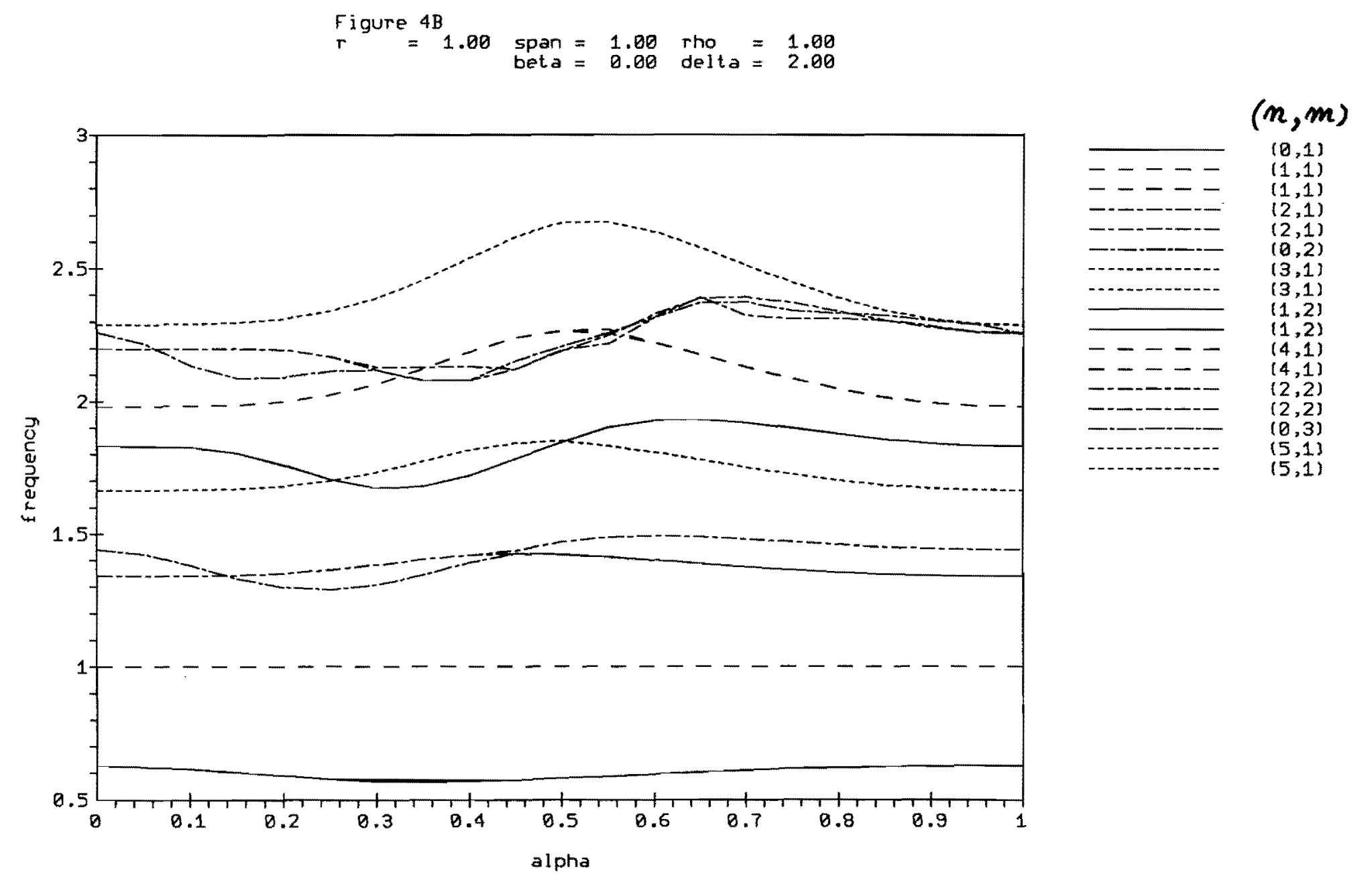




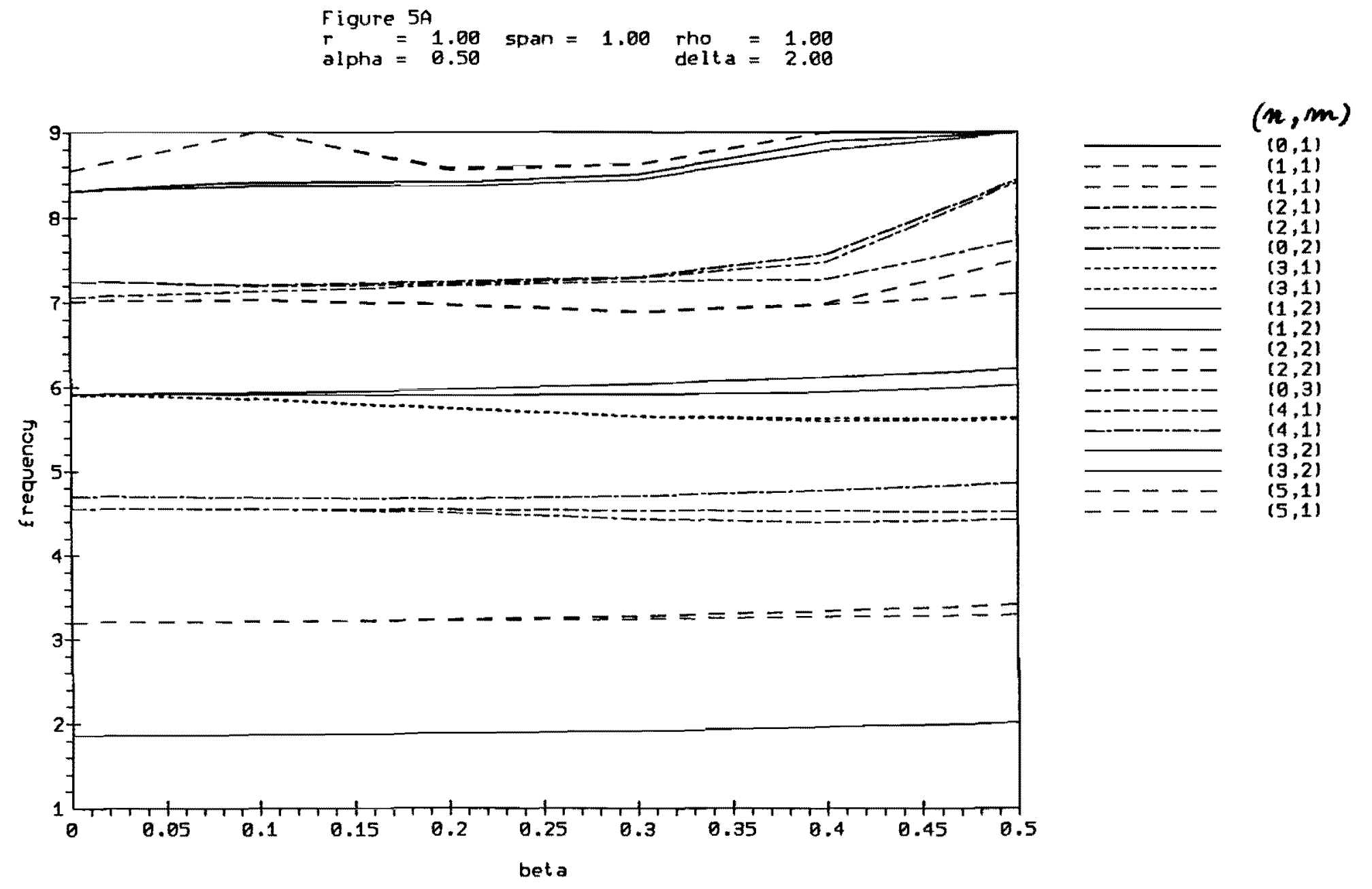




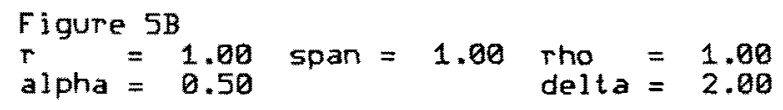

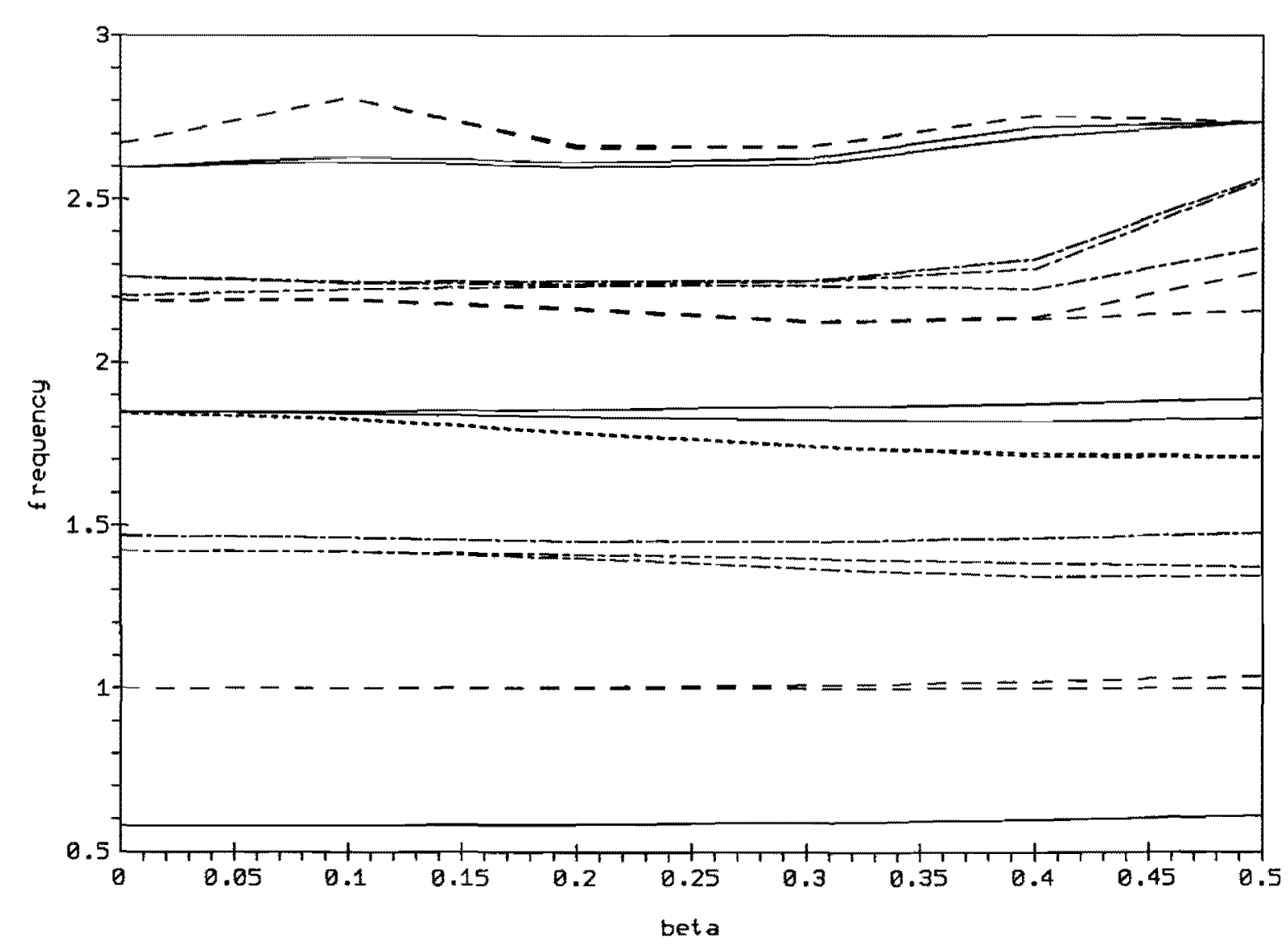

$(n, m)$

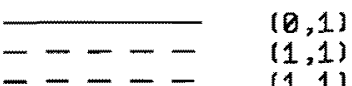
- - - $\quad(1,1)$ \begin{tabular}{l}
$(2,1)$ \\
\hdashline-10
\end{tabular} $\cdots(0,2)$ $(3,1)$
$\cdots \cdots$$\quad(3,1)$ $-(1,2)$ $(2,2)$
$-\ldots-(2,2)$ - - - - - - - - --1$-\begin{array}{r}(3,2) \\ (3,2)\end{array}$ $--\cdots(5,1)$ $-1-z \quad(5,1)$ 
$\begin{array}{lll}\text { Figure } 6 A & & \\ r \quad=1.00 & \text { span }=1.00 \quad \text { rho }=1.00 \\ \text { beta }=0.50 \text { delta }=2.00\end{array}$

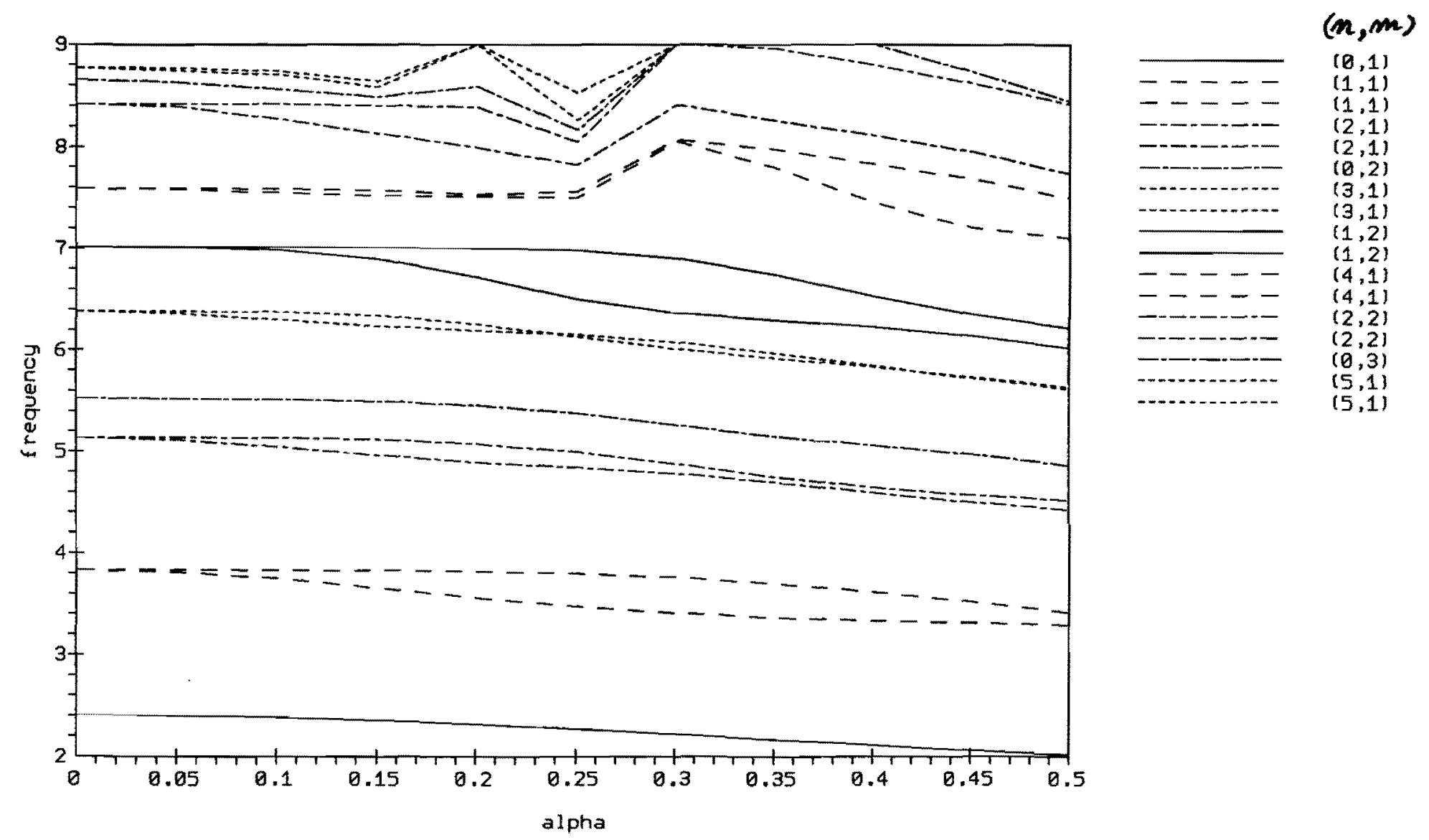




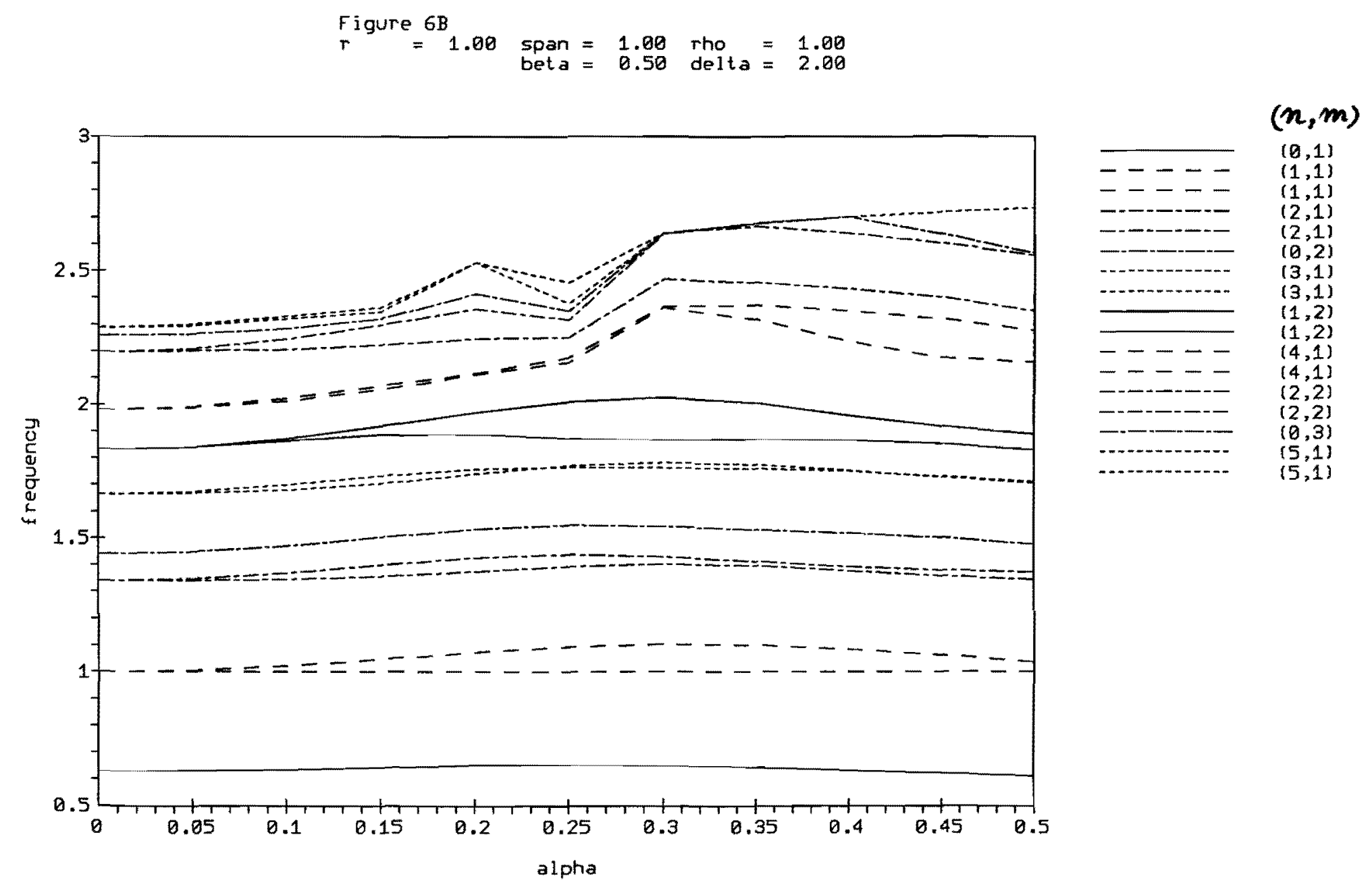


Figure $P A$
$r \quad=1.00$ span $=1.00$ rho $=1.00$
alpha $=0.50$ beta $=0.50$

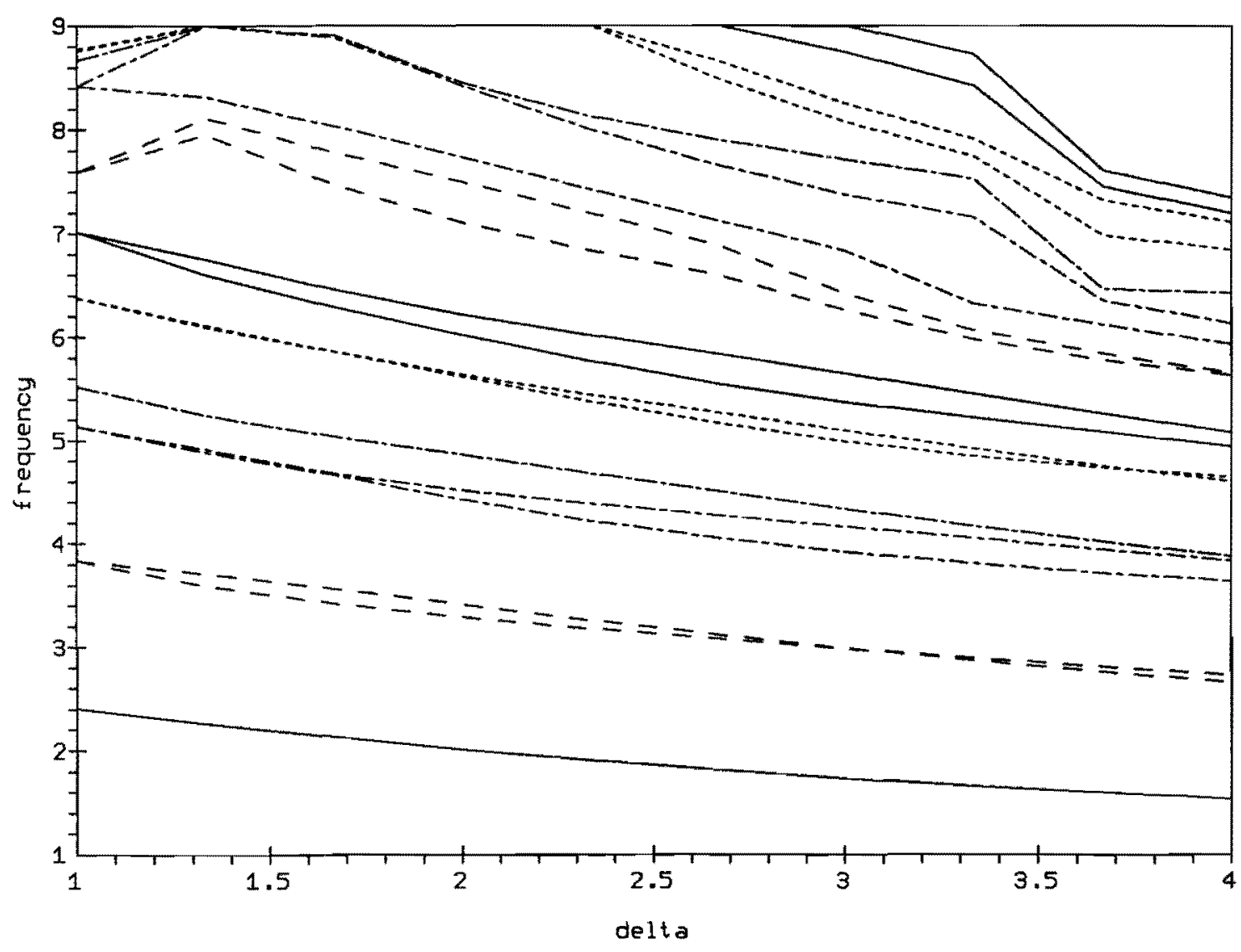

$(n, m)$

$(0,1)$
$--1,1)$

$---2 \quad\left(\frac{1}{2}, 1\right)$

-

- - - - $\quad(0,2)$

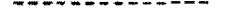

$(3,1)$

$(1,2)$

$(1,2)$

$---(4,1)$

$--\ldots \quad(4,1)$

-......... $(2,2)$

- - - - -

$\ldots \ldots . .15,(5,1)$

$(5,1)$

$(3,2)$ 
Figure $\mathrm{PB}$

$r=1.00$ span $=1.00$ Tho $=1.00$

alpha $=0.50$ beta $=0.50$

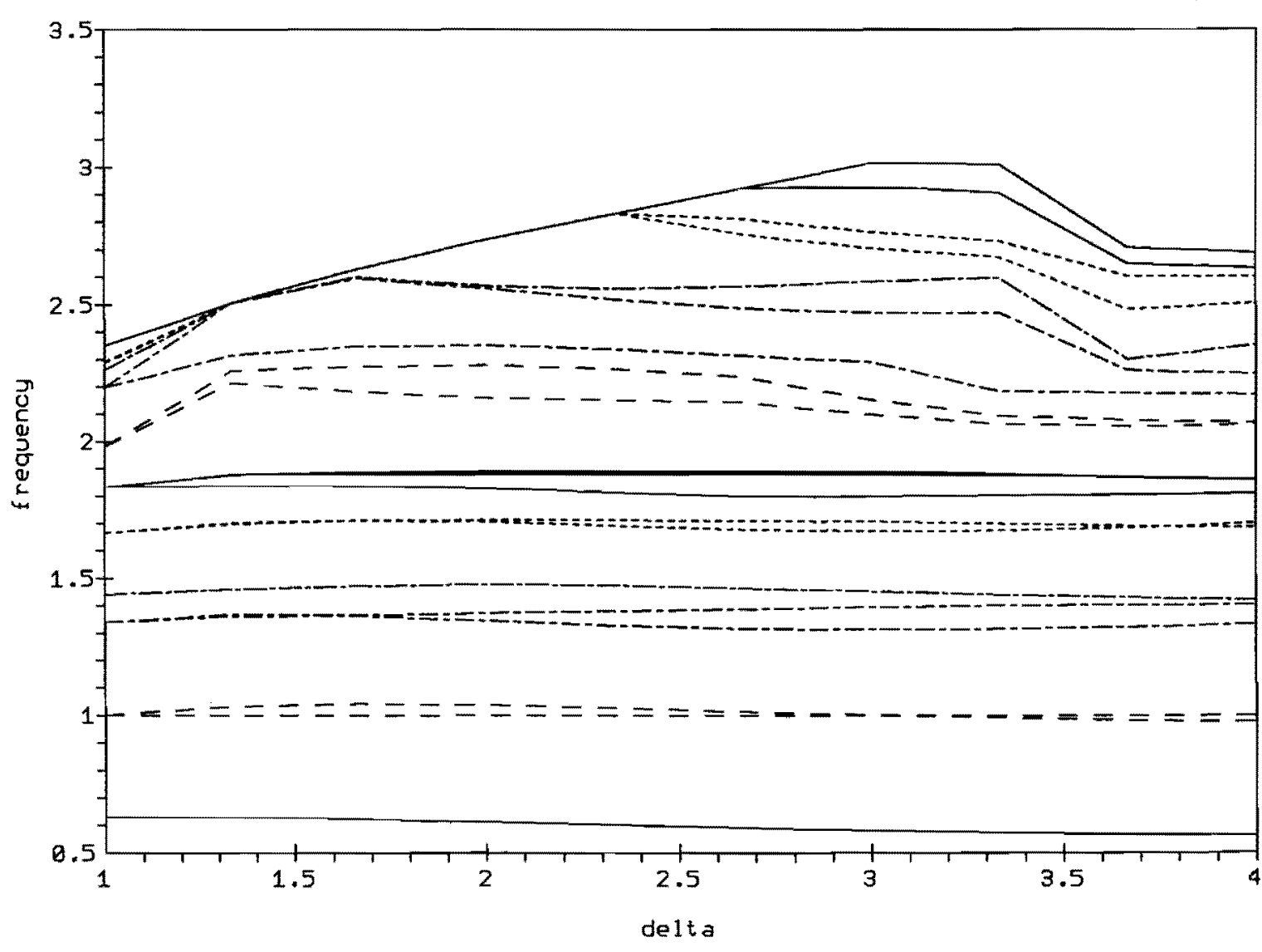

$(n, m)$

$\begin{array}{ll} & (0,1) \\ -\ldots-n & (1,1)\end{array}$

- - - - $(1,1)$

- $-(2,1)$

-

$(3,1)$
$\cdots$

\begin{tabular}{l}
$(3,1\})$ \\
\hline
\end{tabular}

$---1$

$=-(4,1)$

$-\cdots-\cdots \quad(2,2)$

- - - $(0,3)$

$(5,1)$

$(5,1)$

$(3,2)$
$(3,2)$ 